\title{
Heart failure therapy: drugs, genes or stem cells?
}

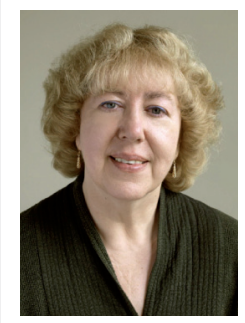

\author{
Sian Harding \\ National Heart \& Lung Institute, Imperial College, Imperial Centre for Translational \\ \& Experimental Medicine, 4th Floor, Hammersmith Campus, Du Cane Road, London, UK \\ sian.harding@imperial.ac.uk \\ “... $\beta$-blockers are ... not just blockers, but are \\ actually activating separate protective pathways \\ in the failing human heart."
}

Professor Sian Harding talks to Caroline Telfer, Assistant Commissioning Editor. Professor Sian Harding obtained her PhD in Pharmacology from King's College, London (UK) in 1981. She became Professor of Cardiac Pharmacology at the National Heart and Lung Institute, a division of the Imperial College Faculty of Medicine, in 2002. Her work has been funded by the British Heart Foundation, the Wellcome Trust, the Medical Research Council, the Biochemical and Biophysical Research Council, the The National Centre for the Replacement, Refinement and Reduction of Animals in Research, Pfizer, GlaxoSmithKline and SmithKline Beecham. Harding is former president of the European Section of the International Society for Heart Research and has organized international cardiovascular science meetings for this society, as well as for the European Society of Cardiology. She is the principal investigator for the first UK gene therapy trial aimed at improving cardiac contractility, organized jointly at Harefield and Papworth Hospitals. Harding is a member of the Nuffield Council on Bioethics and the Medical Research Coucil Regenerative Medicine Research Committee, and Director of a recently awarded British Heart Foundation Cardiovascular Regenerative Medicine Centre. She has been elected Fellow of the American Heart Association, European Society of Cardiology, International Society for Heart Research, Society of Biology and British Society of Pharmacology.

\section{- Can you tell us a little about your career so far?}

I've been at the National Heart and Lung Institute since 1980, when it was the Cardiothoracic Institute. It went through a number of incarnations and is now the National Heart and Lung Institute Division of Imperial College Faculty of Medicine. So I have been in a similar grouping but at different places and in different environments throughout that time. I qualified as a basic scientist in pharmacology (BSc in Pharmacology/Biochemistry and PhD in Cardiac Pharmacology). I targeted the National Heart and Lung Institute and applied to work there even though there was no advertised position. I was taken on as a 5-year post-doctorate with P Harris, then went from there to Lecturer and then all the way through to Professor.

\section{What would you consider to be your greatest achievement to date?}

Neither gene nor cell therapy yet, although I have hopes for both. I think the thing I am most happy about is that I realized and showed that the clinically used $\beta$-blockers are not blockers as such, or not just blockers, but are actually activating separate protective pathways in the failing human heart. I think there is a lot more to be found out about that process and how $\beta$-blockers work in heart failure.

\section{- You mentioned gene therapy. You're the Scientific Principal Investigator for the UK's first clinical trial on myocardial gene therapy. What attracted you to this kind of research?} It was just a natural follow-through really. I wanted to look at the myocytes from failing human hearts because I was interested, then, in the pharmacological mechanisms. I was finding it unsatisfactory to look at animal hearts and then say "I'm doing this because I want to look at human hearts," when I worked at a place where you could get human heart tissue. So I thought it was best to go and try and at least attempt to make some cells or some tissue from those. That's why I went on to develop the myocyte preparation and the human myocyte preparation, in particular. The first question I asked concerned the functional state of the surviving myocytes in the failing human heart. We know that many of these cells are dead, but what about all of the ones that are left? 
Are they performing fantastically well? Or are they performing poorly? If they are not giving optimal force, as we showed, then we have some potential to stimulate them and the overall cardiac output. We, and many other people in the world, found various defects in these myocytes, but one of the things that was common between all causes of heart failure was the change in calcium handling and, particularly, the change in the gene SERCA. It doesn't make a difference whether you have heart disease because of a genetic defect, a heart attack or a valve problem, as this could be a common target.

\section{- How did they discover this SERCA? What research went into this discovery?}

There was a suite of proteins that were known to be involved in moving the calcium around within the cell. SERCA is one of them and it was discovered that the amount of protein was down and the activity was even further decreased because of greater inhibition by other factors. Roger Hajjar, from the Mount Sinai School of Medicine (NY, USA), had developed a way of using viruses to transfect adult myocytes, which are very difficult to genetically manipulate, and had come to me because I knew how to isolate and study human myocytes. Federica del Monte (CardioVascular Institute Beth Israel Deaconess Medical Center, MA, USA), who I still often see, had been my $\mathrm{PhD}$ student and had just the right skills in myocyte experiments and animal models. She went to Roger's laboratory and did much of the work with adenovirus, with my lab contributing from this side. We showed that restoring SERCA changed the failing myocyte behavior into that of normal cells. Most importantly, we showed that it could do it in a way that didn't damage the myocytes, without causing arrhythmias or extra cell death, which a lot of the stimulants do. Therefore, stimulating SERCA seemed to be a good idea. Both Roger and I tried with drug companies to find a way to do this, but nothing useful came out of that (so far). Gene therapy was not the favored option, but Roger took the brave step of using it when no drug was to be found.

- Obviously there are a lot of different conditions that you could target with gene therapy. Why did you choose heart failure in particular? Was it because you had already found this mechanism?

It was really because we knew what we wanted to do and gene therapy was the only way of doing it. Not that we wanted to use gene therapy for anything. In fact, gene therapy is very complex and difficult! If they found a drug now, I think we would be happy.

\section{Are there any leads on that at all?}

There's one that has partly the effect, but the problem is if SERCA is low, especially in endstage heart failure. If levels are very low, you can only get to a certain level by stimulation.

\section{- Can you explain a little about the trial? For example, how many patients were involved and what were the selection criteria?}

CUPID 2 [101] is a follow-on, an expansion of the first CUPID trial [1]. Again, R Hajjar really set this in motion and through the company Celladon (CA, USA), which is now independent, worked out the trial design. That used a small number of patients; approximately 37 patients had the active adeno-associated virus with the SERCA. CUPID showed first that it was safe and second that there was some evidence, although on this very small number of patients, of benefit. CUPID2 is an intermediate trial of 200 patients, through centers in Europe and the USA: the Royal Brompton Hospital is the coordinating centre in the UK, with A Lyon leading it. It is a straightforward percutaneous infusion of one dose of the virus into the coronaries of patients with reasonably severe heart failure. They can recover and go home in a couple of days. There is a second local trial of 24 patients, which we have been trying to get going for quite a while, sponsored by Imperial College. It is funded by the British Heart Foundation, with Celladon contributing the virus and funds for some extra patients. We are using patients who have already had implanted left ventricular assist devices (LVADs). One of the advantages is that it is possible to get tissue from these patients when the pump is implanted and at various times afterwards; for example, if they go to transplant. That will give us information on whether the virus DNA is there and whether the new gene is expressed, in a much more quantitative way. The other thing that is different is that it has some patients who have got neutralizing antibodies to the virus. The natural adeno-associated virus (AAV) is nonpathogenic; it doesn't really give you much of a disease, but many people have been exposed to it, which means that some people won't be eligible for treatment because they have already got antibodies to AAV. 
- Is there any way of getting around this or have you only got the one virus?

We only have one virus. There are potential ways to get over this, such as plasmapheresis, which could wash out the antibodies from the blood beforehand. We have some antibody-positive patients in one arm of the local trial to see exactly how much it will reduce the effect of the virus and just how concerning that might be. This will make a difference to how the treatment can roll out to the whole population.

\section{- How much of the population do you expect to have these positive antibodies?}

Between 30 and 50\%, which is a large percentage. There are other things we can do in the future, such as engineer viruses which have different coat proteins so that they haven't been seen by the immune system. But these are quite complicated things to do.

\section{- In these trials, what kind of end points will you be looking at?}

The first real end point is safety because these are still small trials, so that's the main concern. Then we are looking for some evidence of efficacy in terms of the function of the heart, by imaging and how the patients feel and how far they can walk, and other measures. It gets a little bit more complicated with the patients with LVADs because you have to turn those down or even off to understand the true heart function. But depending on how much tissue we are able to have, we can do something objective in terms of isolating the myocytes from that tissue and looking at their function.

\section{What do you expect will be the outcomes of these trials?}

Well I think that the next outcome will be that there is enough evidence of safety and some evidence of efficacy that will allow Phase III to go ahead.

\section{- We have heard a lot about gene therapy being used in the past and having adverse effects. What steps have been taken this time to make sure that these don't happen?}

This is not a retrovirus, which was the one that caused cancers in a small number of patients in another trial. This virus, AAV, does not integrate into the genome itself (nuclear DNA), so the problems that were encountered with the X-linked severe combined immunodeficiency syndrome trial should not happen because there will be no problem of random integration. Also, the virus itself is inactivated; it does not replicate. So it will deliver its payload, but won't then replicate and grow.

\section{- What do you think will be the progress of gene therapy for heart failure in the next 10 years?}

If this trial goes well then I think it could be a very cost-effective measure since it will be a single-shot application lasting years. It could also be used together with the LVADs to improve the outcome. So I think that it could help patients directly. I also think the fact that you can use it for a nondruggable target might encourage people to think about that option, rather than always trying to go down the drug route. Some things are quite difficult to drug, such as protein-protein interactions, so it might open up a whole new set of targets. I think the LVAD paradigm, where you have patients who are supported by the LVAD and with the added possibility of studying tissue, could be a more useful and safer way of testing out cell therapy eventually.

- You mentioned earlier that you developed the methodology for isolating the individual beating myocytes from the human heart and measuring their function. Can you explain a little more about your research in this area? I thought it was useful to look at the human myocytes. There are pluses and minuses to this. Obviously you can not control what drugs the people are on or when you can get the cells, you can only have end-stage tissue from transplant, and a few surgical samples. You can not do an experiment in the sense of influencing it by giving a patient a drug beforehand. So it is not a replacement for animal work in any way, but it is the real tissue from the real disease, so it is important for that reason. Using myocytes, rather than any other preparation, is important. For example, I could tell that the $\beta$-blocker was having an effect directly on the myocyte and not due to blocking adrenaline. When I put the drugs on the tissue, it might have been blocking something released from intrinsic nerves, so I couldn't really tell unambiguously that the $\beta$-blocker was having an active effect.

\section{- Much of your research focuses on the potential for stem cell therapy in cardiac disease. What have been the milestones of this field and what progress has been made? \\ Firstly, the first wave of bone marrow cell trials. I think that we have been persuaded that there}


is enough benefit, although it is minor, to go on and the big trials will now answer the question one way or another of whether it is worth doing. I think that we're pretty clear that we are not making any new cardiac muscle; it's all a paracrine effect. I think that the endogenous cardiac stem cells coming into those trials are interesting and again it is a little too early to tell, but at least we've got to the point where we can do the trials. I think, in general, the feeling is that there is endogenous repair in the heart and that either by putting some extra cells in or by stimulating it, you could get modest improvements, and it is probably enough to cope with minor damage. It is probably not enough to cope with a very large heart attack and that is the real challenge: how to generate enough actual contracting cardiac muscle and get it to stay in the heart in patients who have got a large amount of cell death. That is one of the things that the new British Heart Foundation Cardiovascular Regenerative Medicine Centre is going to try and contribute to in terms of the field, to actually generate new cardiac muscle, rather than concentrating on the paracrine effect.

\section{- You're heading this center to graft beating heart muscle as part of the British Heart Foundation's Mending Broken Hearts appeal. Can you explain some more about this?}

We are interested in all cells that create new myocytes: by reprogramming or from endogenous cardiac progenitors, or pluripotent stem cells. For grafting, I am looking first at embryonic and induced pluripotent stem cells, particularly because they do very regularly and routinely become cardiac muscle. Certainly more so than bone marrow and more easily than the endogenous progenitor cells. I think tissue engineering is the way forward for cell retention in the heart. Materials, hydrogels, ways of physically stabilizing the muscle, that is probably where the effort needs to go in now. The other thing that is very interesting for me, having worked on human myocytes and struggled with all of the logistical difficulties around that, is the fact that you could use these induced pluripotent stem cell-derived cardiomyocytes as a 'disease in a dish'. That is, a human cardiac myocyte taken from any kind of person, but by making it from a tissue biopsy, making that into stem cells and then making them into myocytes. I think the first information coming out of those things is very very exciting in terms of our understanding of the relationship between the genetic mutation, cell and patient.
- You mentioned induced pluripotent stem cells. Which of the stem cells do you think holds the most promise in therapeutic potential?

I would actually say that I am still very hopeful about the endogenous stem cells and they are clearly going to be easier to get into the clinic. However, it is still very possible that they may not address the problem of large areas of damage. So I think we still have to plough on with the induced pluripotent stem cells. I think that is going to be difficult to do on a patient-by-patient basis: to take somebody's cells, make them into stem cells, make them into cardiac myocytes and then get enough muscle to go back into the patient in a reasonable amount of time.

\section{What kind of timescale are you looking at for this?}

You would have to be waiting 6 months or so. Again we're looking towards the pumps. It may be that you are using this in conjunction with the LVADs, since you can support people on LVADs while you prepare this construct for them. But it does require some changes in the way that the regulation is done because if each of those lines have to be treated as separate products, then it is going to be very difficult logistically, and expensive. It is also difficult to know who is going to pay for it. So the logistics of it may well just torpedo the whole thing.

\section{How do you see the field developing?}

I think that in the next few years, bone marrow cell trials will get larger, until it is decided whether that is a useful strategy. Endogenous stem cell trials will also get larger because the first ones seem to be safe. There will be more standardization on what the cells used actually are and I think that there will be a convergence on what those endogenous cells represent. Then I think there will be a lot of tissue engineering efforts to put generated muscle into the heart. The heart field has a strong experience with device therapy, and tissue engineering is an extension of this. We are quite used to putting foreign material into the heart, with the different implantable cardiac defibrillators, LVAD pumps and cardiac resynchronization devices. One other thing I would say though is that the LVADs seem to be improving. At the moment, they have electrical lines through the skin to an external battery, which makes the patient vulnerable to infection. Now there is work on devices where you can implant the battery and change it every few years. If that works then I think that will probably be a game-changer for the field - maybe we will all be out of a job! 
For more information on the British Heart Foundation 'Mending Broken Hearts Appeal' see their website [102].

\section{Disclaimer}

The opinions expressed in this interview are those of the interviewee and do not necessarily reflect the views of Future Medicine Ltd.

\section{Financial \& competing interests disclosure}

$S$ Harding has received research grants from the British Heart Foundation and Celladon. S Harding has no other relevant affliations or financial involvement with any organization or entity with a financial interest in or financial conflict with the subject matter or materials discussed in the manuscript apart from those disclosed.

No writing assistance was utilized in the production of this manuscript.

\section{References}

1. Jessup M, Greenberg B, Mancini D et al. Calcium Upregulation by Percutaneous Administration of Gene Therapy in Cardiac Disease (CUPID): a Phase 2 trial of intracoronary gene therapy of sarcoplasmic reticulum $\mathrm{Ca} 2^{+}$-ATPase in patients with advanced heart failure. Circulation 124(3), 304-313 (2011).

\section{Websites}

101. A study of genetically targeted enzyme replacement therapy for advanced heart failure (CUPID-2b).

www.clinicaltrials.gov/show/ NCT01643330

102. British Heart Foundation Mending Broken Hearts Appeal. www.bhf.org.uk/mbh 